\title{
Stochastic thermodynamics of nanoscale friction
}

\author{
P. C. Torche, ${ }^{1, \text { * }}$ P. Nicolini, ${ }^{2}$ T. Polcar, ${ }^{1}$ and O. Hovorka ${ }^{1}$ \\ ${ }^{1}$ Engineering and Physical Sciences, University of Southampton, Southampton, UK \\ ${ }^{2}$ Department of Control Engineering, Faculty of Electrical Engineering, \\ Czech Technical University in Prague, Prague 2, Czech Republic
}

(Dated: July 11, 2021)

\begin{abstract}
Developing the thermodynamics of nanoscale friction is needed in a wide range of tribological applications, where the key objective is to optimally control the energy dissipation. Here we show that the modern stochastic thermodynamics allows interpreting the measurements obtained by the friction force microscopy, which is the standard tool for investigating frictional properties of materials, in terms of basic thermodynamics concepts such as the fluctuating work and entropy. We show that this allows the identification of the heat produced during the friction process as an unambiguous measure of thermodynamic irreversibility. We have applied this procedure to quantify the heat produced during the frictional sliding in a broad velocity range, and observe velocity-dependent scaling behaviour, which is useful for interpreting the experimental outcomes.
\end{abstract}

\section{INTRODUCTION}

Recent decades are witnessing a rapid development of out-of-equilibrium thermodynamics of small fluctuating systems to minimise energy losses in applications. For example, optimising the heat dissipation occurring during nanoscale friction processes is of paramount importance for the bottom-up tribological material design [1]. This is a challenge because friction is a far-from-equilibrium phenomenon and its fully consistent thermodynamic description falls beyond the validity range of non-linear thermodynamics [2] or linear response theory [3]. In particular, the general principles governing the separation of thermodynamic work into the system's internal free energy and entropy or heat produced during the friction process are currently unknown, especially at the nanoscale dominated by thermal and structural fluctuations.

In this article the aim is to address these questions by adapting the modern stochastic thermodynamics (ST) [4] to nanoscale friction. ST is a fully consistent general thermodynamic theory of irreversible processes applicable to thermally fluctuating mesoscopic systems, and defines the essential thermodynamic concepts such as the fluctuating work, free energy, and entropy [5, 6]. Its practical application to nanoscale friction requires formulating these concepts in terms of the state variables relevant to thermally activated friction processes quantifiable by the friction force microscopy (FFM), which is the key experimental tool used to study nanoscale friction [7 15].

The output of FFM is a randomised signal reflecting the structure of atomic interactions between the FFM tip and the material, and carries complete information about the underlying thermodynamic irreversibility. It frequently requires interpretation based on detailed atomistic models combining the molecular dynamics with force-fields obtained from $a b$ initio methods [16, 17]. However, the atomistic modelling techniques are often practically limited by their computational scalability to mesoscopic length and time-scales of a typical FFM experiment, and instead adopted are often non- linear coarse-grained approaches based on the PrandtlTomlinson [18, 19] or Frenkel-Kontrova 20] models. This 'mesoscopic' level of modelling is therefore typically adopted for studying the thermodynamics aspects of nanoscale friction [21 23].

In this work, we considered the classical PrandtlTomlinson model combined with Kramers transition state theory of thermal fluctuations 24 as a model of single asperity friction. We quantified the energy barrier distributions, and assembled the master-equation governing the thermally activated dynamics using the standard approach [21, 23, 25, 27]. Solving the thermally activated dynamics allowed evaluating the time-dependent probability distributions and formulating the expressions for fluctuating entropy based on ST [4, and express the first thermodynamic law in terms of the fluctuating work and heat produced during the frictional sliding 28]. We adopted the Crooks and Jarzynski fluctuation relations of ST [29, 30] to establish the relationship between the work and the internal system free energies, similarly to earlier work [21, 22]. Then we showed that these relations can be used to predict the entropy or heat production from practical FFM measurements. Finally, we developed an approach for estimating the heat production from velocity-dependent friction measurements by identifying new empirical scaling relation, which extends the existing family of such relations [12, 31,35.

\section{MODEL OF FRICTION}

To model a typical FFM experiment of a thermally activated stick-slip friction process we consider the singleasperity Prandtl-Tomlinson model with thermal fluctuations included via transition state theory [33]. A sharp tip connected by a spring to the FFM cantilever is sliding with velocity $V$ along a static substrate in the $X$ direction (Fig. 11(a)). The potential energy is defined 
(a)
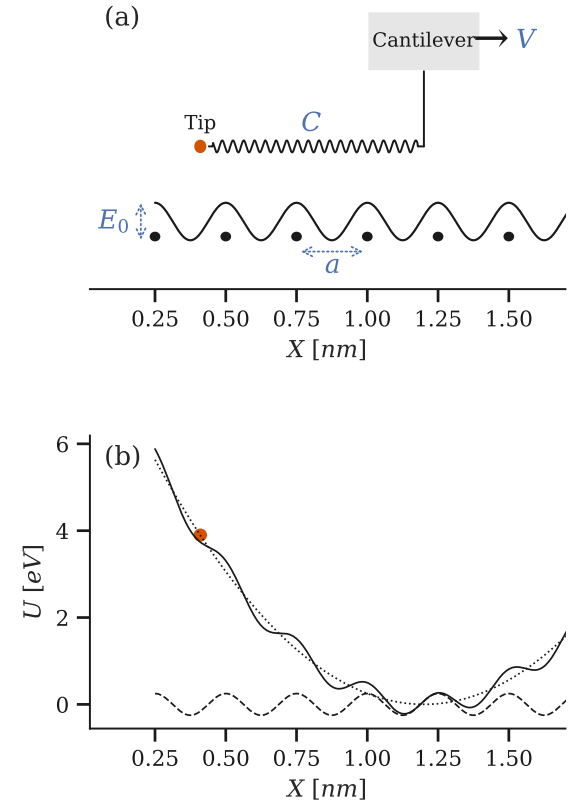

Figure 1. (a) A sketch of the one-dimensional PrandtlTomlinson model of FFM experiments with $C=2 \mathrm{~N} / \mathrm{m}$, $V t=1.2 \mathrm{~nm}, a=0.25 \mathrm{~nm}$, and $E_{0}=0.5 \mathrm{eV}$, in the range of typical values for FFM experiments [36]. (b) shows the total energy landscape (continuous line), the substrate-tip interaction term (dashed line), and the elastic term (dotted line). The instantaneous position of the tip in this scenario is $x \approx 0.4 \mathrm{~nm}$, and energy $u \approx 4 \mathrm{eV}$.

as:

$$
U(X, t)=-\frac{E_{0}}{2} \cos \left(\frac{2 \pi X}{a}\right)+\frac{C}{2}(X-V t)^{2}
$$

The first term in Eq. (1) is the energy of the substrate-tip interaction of strength $E_{0}$, and $a$ is the spacing between the surface asperities. The second term corresponds to the elastic energy of the cantilever, where $C$ is the elastic energy constant and the product $V t$ is the position of the cantilever at time $t$. An example of the energy profile is shown in Fig. 1(b). The total force acting on the tip is $F_{\text {tot }}(X, t)=-\partial U / \partial X=F_{s}(X)+F(X, t)$, where the interaction force is $F_{s}(X)=-\left(\pi E_{0} / a\right) \sin (2 \pi X / a)$, and the elastic force exerted on the tip by the cantilever reads:

$$
F(X, t)=-C(X-V t)
$$

This definition of the elastic force is typically used to represent the friction force in FFM. The energy minima at any time $t$ in Eq. (1) define the system states, $n$, corresponding to stable tip positions $X_{n}$. Correspondingly, the energy of a state $n$ is $U_{n}(t)=U\left(X_{n}\right)$. Due to the explicit time dependence in the second term in Eq. (1), the energy landscape and both $X_{n}$ and $U_{n}$ evolve during the sliding motion with a frequency $V / a$. It can be shown that the necessary condition for the model to represent the stick-slip motion is $(2 \pi)^{2} E_{0} / C a^{2} \geq 1$ 7, 12, 37, as otherwise only one energy minimum is available at all times leading to continuous sliding [38, 39].

Thermal activation is viewed as a Markovian random hopping process over energy barriers $\Delta U_{m n}$ separating the different states $m$ and $n$. The rate of thermally activated transitions from state $n$ to $m$ is given by the Arrhenius law 33]:

$$
\omega_{m n}=\omega_{0} \exp \left(-\beta \Delta U_{m n}\right)
$$

where $\omega_{0}$ is the attempt frequency setting the characteristic timescale of thermal fluctuations, and $\beta=1 /\left(k_{B} T\right)$ with $T$ being the temperature and $k_{B}$ the Boltzmann constant. Note that due to the explicit time-dependence in the elastic term in Eq. (1) both $\Delta U_{m n}$ and $\omega_{m n}$ are time-dependent.

The time evolution of the system is fully determined by solving the master equation:

$$
\frac{d P_{m}}{d t}=\sum_{n}\left(\omega_{m n} P_{n}-\omega_{n m} P_{m}\right)
$$

which allows obtaining the distribution of probabilities $P_{n}(t)$ that the system resides in the state $n$ at the time $t$. Eq. (4) represents a set of coupled ordinary differential equations with initial conditions $P_{n}(t=0)$ for all $n$, which can be solved by standard numerical techniques [40. An alternative way of solving Eq. (4) is by generating the individual randomised trajectories of the state variables by using kinetic Monte Carlo methods (kMC), such as the so-called fixed time-step kMC [41]. Both methods are used in this work. To implement these methods, we considered only the transitions occurring between the immediately neighbouring states, i.e. the transitions from $n$ to $m$ where $m=n-1$ or $n+1$, which allowed determining the corresponding energy barriers required to evaluate the transition rates in Eq. (3). This assumption is justifiable for over-damped systems when the thermally activated transitions between distant states are expected to be statistically rare [23].

Finally, note that in the limit of high energy barriers and large damping, the present master equation approach becomes equivalent to the frequently used stochastic Langevin dynamics, and is often adopted for studying the thermally activated processes in a typical FFM experiment for the sake of computational convenience [42].

\section{THERMODYNAMICS OF STOCHASTIC FRICTION PROCESSES}

We have used the kMC to compute random sequences of states $n(t)$ in the time interval from $t=0$ to $t=$ $t_{f}$. Each sequence $n(t)$ defines a particular path in the state space. The corresponding trajectory of the tip, $x(t)$, can be obtained as a series of tip positions $X_{n}$ along $n(t)$. The lowercase notation $x(t)$ is used to emphasise the path-dependent stochastic process rather than the 

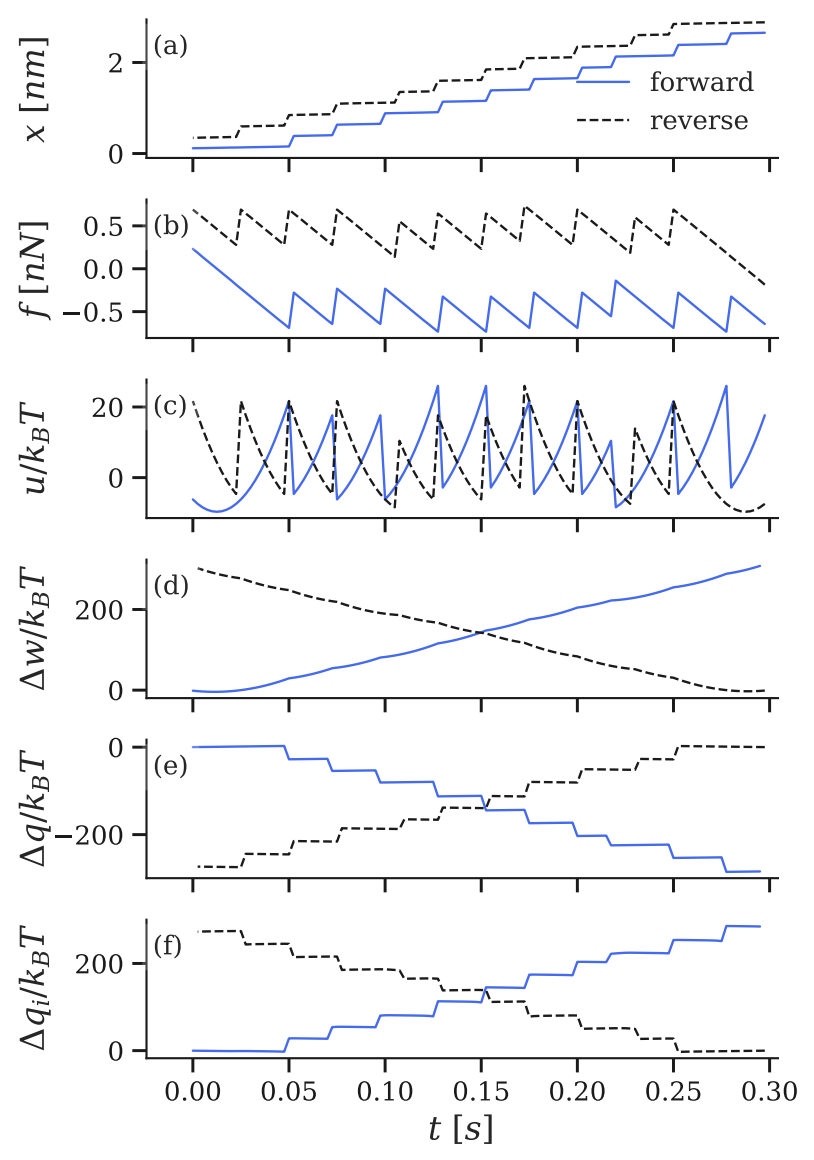

Figure 2. Kinetic Monte-Carlo simulation based on Eqs. (1)-(4) of thermodynamics of a FFM tip subject to forward (solid line) and reversed driving (dotted line). Shown are the stochastic processes for (a) tip position, (b) friction force, and cumulative changes of (c) internal energy, (d) work, (e) heat exchanged with the environment, and (f) heat produced during the sliding process. The forward process starts at time $t=0$ with the cantilever at position $x=0$ and the tip in thermal equilibrium, and follows the driving protocol $\lambda=V t$ until the finite time $t=t_{f}$. The reverse process starts in thermal equilibrium at time $t_{f}$, with the cantilever at position $x=V t_{f}$ and follows the protocol $\lambda=V t_{f}-V t$ from $t=0$ until $t=t_{f}$. All cumulative changes $\Delta$ are calculated by summing up all the time contributions, subject to resetting the counter at $t_{f}$. Simulation parameters fixed as in Fig. 1 , with $V=10 \mathrm{~nm} / \mathrm{s}, T=300 \mathrm{~K}$, and $\omega_{0}=20 \mathrm{kHz}$.

state variables $X_{n}$. Then, we evaluated the fluctuating friction force $f(t)$ by inserting $x(t)$ in Eq. (2).

Figs. 2(a) and (b) show examples of sequences $x(t)$ and $f(t)$. The randomised character of these processes is due to thermal fluctuations at finite temperature. The solid line corresponds to the forward tip sliding process with velocity $V$, which starts in thermal equilibrium at the initial time $t=0$ and progresses until the final time $t_{f}$. The dotted line corresponds to the reverse process. The reverse process also starts in thermal equilibrium, obtained by allowing the system to equilibrate after stopping the forward process at $t=t_{f}$ and then resetting the clock. Thus the small gap between the end-point of the forward process and the starting point of the reverse process seen at $t=t_{f}$ in Fig. 2(a) corresponds to thermal relaxation towards the new equilibrium state.

Fig. 2(c) shows the fluctuating energy $u(t)$ evaluated by applying Eq. (1) over the forward and reverse trajectories $x(t)$. Expressing the total time derivative over the path $n(t)$ associated with $x(t)$ gives:

$$
\frac{d u}{d t}=\frac{\partial u}{\partial t}+\frac{\partial u}{\partial n} \frac{d n}{d t}
$$

The first partial derivative can be evaluated by directly differentiating Eq. (1), and since only its second term contains explicit time-dependence we obtain:

$$
\frac{\partial u}{\partial t}=-C V(x-V t)=V f(t) \equiv \frac{d w}{d t}
$$

where we used Eq. (2) to express the fluctuating friction force $f(t)$ associated with the tip trajectory $x(t)$. The product of the velocity and friction force is the work per unit time performed by the FFM tip on the system. Thus Eq. (6) is the definition of fluctuating work $w$ associated with the friction process. Fig. 2(d) shows an example of the work $\Delta w$ accumulated during the forward and reversed sliding processes.

To express the second term in Eq. (5), consider a particular thermally activated jump from a state $n=n^{-}$ to a new state $m=n^{+}$occurring at the time instant $t_{j}$. Then the derivative $\partial u /\left.\partial n\right|_{t=t_{j}}=\lim _{\Delta n \rightarrow 0}(u(n+\Delta n)-$ $u(n)) /\left.\Delta n\right|_{t=t_{j}}=\left(U_{n_{+}}\left(t_{j}\right)-U_{n_{-}}\left(t_{j}\right)\right) /\left(n_{+}\left(t_{j}\right)-n_{-}\left(t_{j}\right)\right)$, which is simply the ratio of the energy change over the state change associated with the thermally activated transition at $t_{j}$. Similarly, we can express the time derivative of the state-space path as $d n /\left.d t\right|_{t=t_{j}}=$ $\lim _{\Delta t \rightarrow 0}\left(n\left(t_{j}+\Delta t\right)-n\left(t_{j}\right)\right) / \Delta t=\delta\left(t-t_{j}\right)\left(n_{+}\left(t_{j}\right)-\right.$ $\left.n_{-}\left(t_{j}\right)\right)$, where the factor $\Delta t$ was merged into the definition of the Dirac delta function $\delta(t)$. Combining both results we obtain for the second term appearing in Eq. (5) the expression $(\partial u / \partial n)(d n / d t)=\delta\left(t-t_{j}\right)\left(U_{n_{+}}\left(t_{j}\right)-\right.$ $\left.\sigma_{n_{-}}\left(t_{j}\right)\right)$. This expression can be arranged further by realising that since $U_{n_{-}}$and $U_{n_{+}}$are valid state energies corresponding to states $n_{-}$and $n_{+}$, their energy difference can be related to the transition rates through the use of Eq. (3) as $U_{n_{+}}\left(t_{j}\right)-U_{n_{-}}\left(t_{j}\right)=$ $k_{B} T \ln \left(\omega_{n_{-} n_{+}}\left(t_{j}\right) / \omega_{n_{+} n_{-}}\left(t_{j}\right)\right)$. Inserting this expression into the time-derivative term obtained above and arranging gives:

$$
\frac{\partial u}{\partial n} \frac{d n}{d t}=k_{B} T \sum_{j} \delta\left(t-t_{j}\right) \ln \frac{\omega_{n_{-} n_{+}}\left(t_{j}\right)}{\omega_{n_{+} n_{-}}\left(t_{j}\right)} .
$$

Note that the summation over the time instants $t_{j}$ follows from repeatedly applying the above differentiation procedure to all thermally activated jumps occurring along the path $n(t)$.

To understand the meaning of the result in Eq. (7), it is first necessary to introduce the notion of the fluctuating 
(stochastic) entropy over the path $n(t)$ by following the earlier work [4, 29]:

$$
s(t)=-k_{B} \ln P_{n(t)}(t) .
$$

According to this expression, evaluating the stochastic entropy requires explicitly solving the master-equation Eq. (4) to obtain the time-dependent state probabilities $P_{n}(t)$, and aligning the solutions in time with each particular realisation of the stochastic path $n(t)$ generated by the kinetic Monte-Carlo method. Differentiating Eq. (8) with respect to time, by using a procedure similar to that used above for Eq. (7), we obtain:

$$
\frac{d s}{d t}=-k_{B} \frac{\partial_{t} P_{n(t)}}{P_{n(t)}}-k_{B} \sum_{j} \delta\left(t-t_{j}\right) \ln \frac{P_{n_{+}}\left(t_{j}\right)}{P_{n_{-}}\left(t_{j}\right)},
$$

where the symbol $\partial_{t} \equiv \partial / \partial t$. The first term describes the contribution to the total entropy change from the smooth variation of state probabilities. The second term describes the contribution from the instantaneous jumps between the states occurring during thermally activated transitions at random time instants $t_{j}$.

Following the standard non-equilibrium thermodynamics 2, it is useful to split the total entropy change into the entropy flow and entropy production:

$$
\frac{d s}{d t}=\frac{d_{e} s}{d t}+\frac{d_{i} s}{d t}
$$

Earlier work 4, 43 introduced explicit expressions for stochastic entropy production:

$$
\begin{aligned}
\frac{d_{i} s}{d t}= & -k_{B} \frac{\partial_{t} P_{n(t)}}{P_{n(t)}} \\
& -k_{B} \sum_{j} \delta\left(t-t_{j}\right) \ln \frac{P_{n_{+}}\left(t_{j}\right) \omega_{n_{-} n_{+}}\left(t_{j}\right)}{P_{n_{-}}\left(t_{j}\right) \omega_{n_{+} n_{-}}\left(t_{j}\right)},
\end{aligned}
$$

and entropy flow:

$$
\frac{d_{e} s}{d t}=k_{B} \sum_{j} \delta\left(t-t_{j}\right) \ln \frac{\omega_{n_{-} n_{+}}\left(t_{j}\right)}{\omega_{n_{+} n_{-}}\left(t_{j}\right)}
$$

such that Eqs. (9) and (10) are satisfied. These definitions are consistent with their ensemble-averaged equivalents 4, 23.

Given the definitions above we observe that Eq. (12) in fact recovers Eq. (7). Inserting Eqs. (6), (7), and (12) into Eq. (5) and arranging, we obtain:

$$
\frac{d u}{d t}=\frac{d w}{d t}+T \frac{d_{e} s}{d t}
$$

This relation establishes the first thermodynamic law for the friction process. It represents the energy balance valid over the fluctuating trajectory of a FFM tip, relating the changes of the internal energy change of the system to the work and heat exchanged with the environment along the fluctuating trajectory. The notion of the fluctuating heat can be made explicit in analogy with Carnot-Clausius theorem [2]:

$$
\frac{d q}{d t}=T \frac{d_{e} s}{d t}
$$

which upon inserting into Eq. (13) gives the familiar form of the first thermodynamic law:

$$
\frac{d u}{d t}=\frac{d w}{d t}+\frac{d q}{d t}
$$

Eq. (15) affirms the interpretation of Eq. (12) as the entropy flow and, in fact, verifies that the definitions of variables as given above are consistent with the first law of thermodynamics.

Finally, the entropy production in Eq. (11) quantifies the extent of irreversible processes occurring within the system. It is a measure of irreversible heat:

$$
\frac{d q_{i}}{d t}=T \frac{d_{i} s}{d t}
$$

produced during the friction process. This is in general different from work, which is related to the friction force according to Eq. (6). Therefore, quantifying the dissipative processes by using the friction force, as is often done in practice, may often be insufficient and it may be necessary to determine the entropy (heat) production directly. Fig. 2(e)-(f) show examples of cumulative heat exchanged with the heat bath and the heat produced in the system. Their sum gives the total fluctuating entropy change $\Delta s$, which is on the order of $\Delta s / k_{B} \approx 3$ and thus rather small, making the plots in Fig. 2(e) and (f) appear indistinguishable on the scale chosen apart from the reversed trend.

\section{ESTIMATION OF ENTROPY PRODUCTION}

Repeated FFM scans produce statistical variation in the fluctuating trajectories as illustrated in Fig. 3. The figure shows the calculations over the ensemble of stochastic processes statistically equivalent to Fig. 2. The mean values of the various thermodynamic variables are shown by solid lines and obey the ensemble thermodynamics of friction developed earlier 23. The spread around the mean corresponds to the standard deviation of thermal fluctuations observed along the individual paths. As it turns out, these fluctuations can be used to estimate the free energy and entropy production from practically realisable FFM measurements of the fluctuating work. We demonstrate this by considering the Crooks and Jarzynski fluctuation relations [29, 30].

The Crooks fluctuation relation [29] states the relationship between the measurements of the cumulative fluctuating work $\Delta w$ expended in a non-equilibrium process occurring between two thermodynamic equilibrium states attained at $t=0$ and $t=t_{f}$, and the associated free energy difference $\Delta \mathcal{F}$ between these equilibrium states. Let 

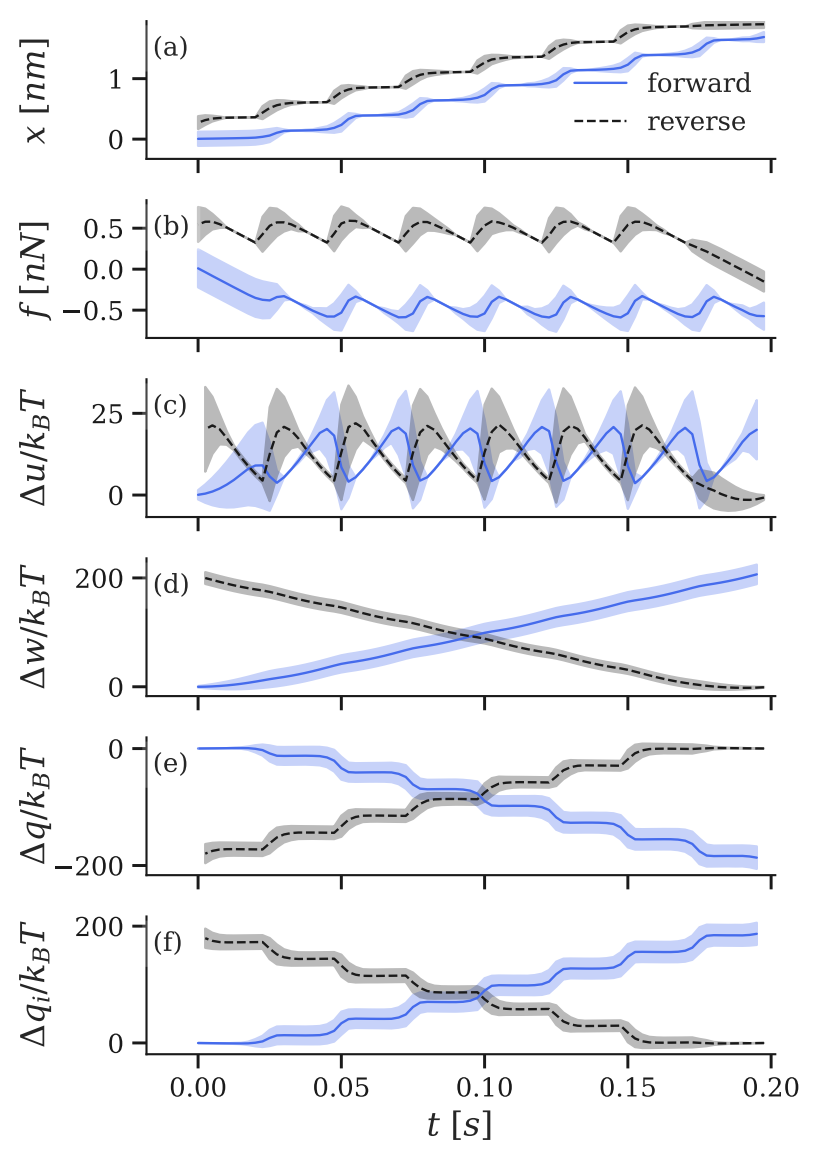

Figure 3. A plot analogous to Fig. 2 containing 500 individual trajectories. The lines are the mean values obtained over the 500 trajectories, and the shaded areas imply two standard deviations around the mean.

$\mathcal{P}$ be the probability distribution to observe $\Delta w$, and $\mathcal{P}_{R}$ the probability distribution of the equivalent work $-\Delta w$ observed when the system is driven in a reversed manner. Then it can be shown that for microscopically reversible fluctuating dynamics, which is obeyed by our model introduced in Sec. III, the following relation holds:

$$
\frac{\mathcal{P}(+\Delta w)}{\mathcal{P}_{R}(-\Delta w)}=e^{-\beta \Delta w+\beta \Delta \mathcal{F}}
$$

This relation allows determining the equilibrium free energy difference $\Delta \mathcal{F}$ from the measurements of the nonequilibrium fluctuating work $\Delta w$.

To demonstrate this, we generated a large set of the forward and reverse friction processes for a broad range of model parameters using the procedure introduced above (Fig. 2). The equilibrium probability distribution of the initial state at $t=0$ was determined from the Boltzmann distribution. We took advantage of periodicity inherent in Eq. (1) and set the value of $t_{f}$ to correspond to a length multiple of the lattice constant, such that the free energy of the new equilibrium state is the same as that
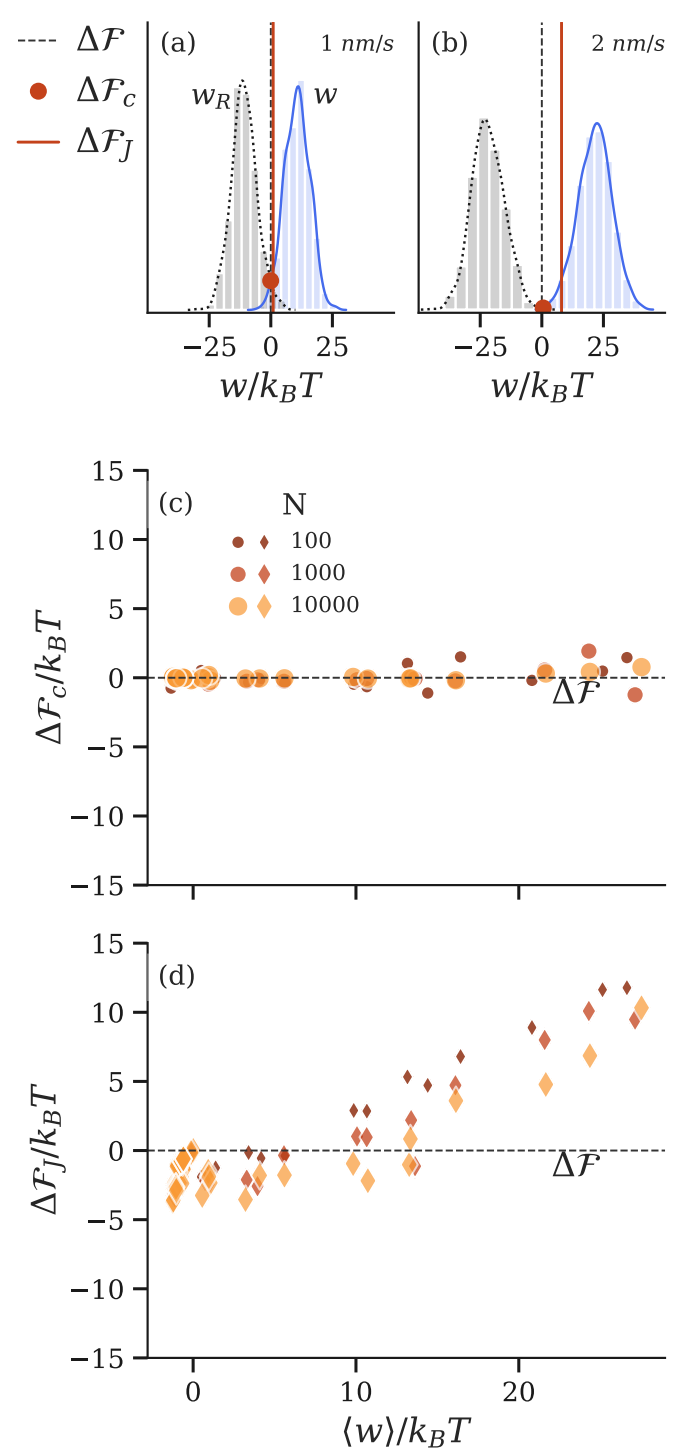

Figure 4. (a)-(b) Examples of probability distributions of cumulative fluctuating work for the forward $(\mathcal{P})$ and reverse process $\left(\mathcal{P}_{R}\right)$ obtained as histograms over 1000 different trajectories for velocities $V=1$ and $2 \mathrm{~nm} / \mathrm{s}, C=9 \mathrm{~N} / \mathrm{m}, \omega_{0}=100$ $\mathrm{kHz}$, and the rest of parameters fixed as in Fig. 2. The estimates using the Crooks $\left(\Delta \mathcal{F}_{c}\right)$ and Jarzynski relations $\left(\Delta \mathcal{F}_{J}\right)$ are shown for comparison. (c)-(d) The free energy difference estimated from the Crooks and Jarzynski relations as a function of the mean fluctuating work for a variable number $N$ of repeated fluctuating trajectories corresponding to $C$ in the range $5-9 \mathrm{~N} / \mathrm{m}, E_{0}$ between $0.3-0.7 \mathrm{eV}, V$ between $0.5-2$ $\mathrm{nm} / \mathrm{s}, T$ in the range $300-500 \mathrm{~K}$, fixed $a=0.25 \mathrm{~nm}$ and $\omega_{0}=100 \mathrm{kHz}$. The dashed line in all plots corresponds to exact reference free energy $\Delta \mathcal{F}=0$.

of the initial state, i.e. $\Delta \mathcal{F}=0$. The cumulative fluctuating work $\Delta w$ expended during the time interval was calculated using Eq. (6).

Figs. 4(a) and (b) show examples of the calculated probability distributions $\mathcal{P}(\Delta w)$ and $\mathcal{P}_{R}(-\Delta w)$ for two 
different sliding velocities. The distributions $\mathcal{P}$ and $\mathcal{P}_{R}$ show a significant spread around the mean value, consistently with Fig. 3. Both distributions cross at the work value $\Delta w_{c}$ when $\mathcal{P}\left(\Delta w_{c}\right) / \mathcal{P}_{R}\left(-\Delta w_{c}\right)=1$. According to Eq. 177 this implies that $\Delta w_{c}=\Delta \mathcal{F}_{c}$, where the subscript $c$ means the estimate is based on the Crooks relation. This value is highlighted in Figs. 4(a)-(b) by the dot estimating $\Delta \mathcal{F}_{c}=0$, as expected. Fig. 4 (c) shows a further validation of Eq. (17), where $\Delta \mathcal{F}_{c}$ was estimated based on histograms of a variable number $N$ of fluctuating trajectories. This plot illustrates that the precision increases with increasing $N$. Thus, repeated FFM measurements of the fluctuating work allow mapping the free energy profile across the sample surface.

Jarzynski fluctuation relation 30 is related to Eq. (17) and can be expressed as:

$$
\left\langle\mathrm{e}^{-\beta \Delta w}\right\rangle=\mathrm{e}^{-\beta \Delta F} .
$$

The symbol $\langle\ldots\rangle$ implies averaging over many trajectories again starting and ending in thermally equilibrated states. Similar to Eq. (17), Eq. (18) allows determining the equilibrium free energy difference from a set of measurements of fluctuating work in a series of FFM scans, with the advantage that it relies only on the forward trajectories. The disadvantage is in the increased statistical error. This can be seen in Figs. 4(a)-(b) showing reduced accuracy of $\Delta \mathcal{F}_{J}$, where $\Delta \mathcal{F}_{J}$ denotes the estimate of the true free energy $\Delta \mathcal{F}$ using the Jarzynski relation, which becomes more pronounced with increasing sliding velocity. Fig. $4\left(\right.$ d) shows the estimates of $\Delta \mathcal{F}_{J}$ for a variable number $N$ of fluctuating trajectories, demonstrating that although the deviating trend of the free energy estimates seen for larger work $\Delta w$ improves with increasing $N$, significantly more measurements are required to improve the accuracy further. This observation is consistent with the earlier findings 22, which suggested that the application of Eq. [18) is practical for work-temperature ratios up to $\langle\Delta w\rangle / k_{B} T \approx 1$. For $\langle\Delta w\rangle / k_{B} T>>1$ the accuracy suffers from the occurrence of rare trajectories of large statistical significance, observing which requires an exponentially increasing number $N$ of measurements.

We also use the developed framework to quantify entropy production which is a direct measure of thermodynamic irreversibility. The entropy production $\Delta_{i} s$ can be computed directly by integrating Eq. (11) over a fluctuating trajectory, which in practical applications requires fitting the model to the measurement data. It turns out the $\Delta_{i} s$ can be estimated from measurements directly with aid of Crooks or Jarzynski relations. To see this, we first recall the thermodynamic relation relating the free energy to entropy production $\Delta \mathcal{F}=\Delta w+T \Delta_{i} s$, which holds for non-equilibrium isothermal processes at constant temperature $T$ of the heat bath [4. If the forward process starts and ends in thermal equilibrium, their equilibrium free energy difference $\Delta \mathcal{F}$ can be estimated from practical measurements using the Crooks or Jarzynski relations as discussed above. Then the entropy production can be related to the fluctuating work mea-

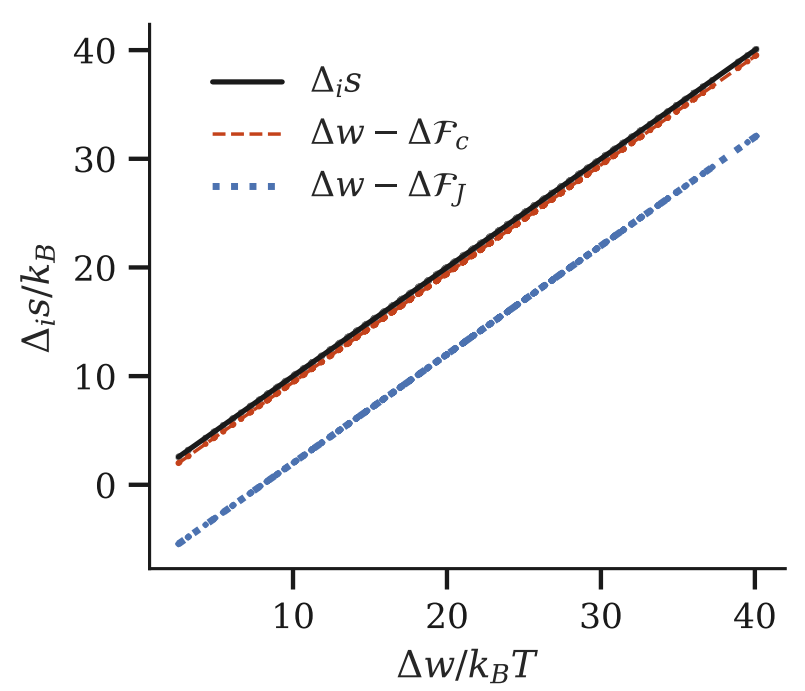

Figure 5. A plot of an estimate of the cumulative entropy production $T \Delta_{i} s$, or equivalently the heat $\Delta q_{i}$ according to Eq. 16, as a function of the cumulative fluctuating work. The exact value (black, solid line) is found using the stochastic thermodynamics developed in Sec. III] The estimations are based on Eq. (19) using the Crooks (red, dashed line) and Jarzynski relations (blue, dotted line) to obtain the free energy $\Delta \mathcal{F}$. Simulation data equivalent to Fig. 4(b).

sured over the trajectory simply by the linear relation:

$$
T \Delta_{i} s=\Delta \mathcal{F}-\Delta w
$$

Note that in this context, the entropy production can be see as the heat produced during the friction process expressed through Eq. (16) as $\Delta q_{i}=T \Delta_{i} s$.

Fig. 5 compares the entropy production for the data set in Fig. 4(b) evaluated based on the exact stochastic thermodynamics formalism developed in Sec. III, used here as benchmark, and entropy production estimate based on Eq. (19) with the $\Delta \mathcal{F}$ approximated by $\Delta \mathcal{F}_{c}$ and $\Delta \mathcal{F}_{J}$ as discussed above. Clearly, the accuracy of prediction of the entropy production depends on the accuracy of estimate of the free energy, and Crooks relation is more accurate as expected. Thus, once the free energy difference has been obtained with sufficient accuracy, any measurement of the fluctuating work $\Delta w$ can be converted to the associated entropy production $\Delta_{i} s$.

It is worthwhile mentioning that dissipation in the practical nanoscale friction experiments is often evaluated based on the cyclic FFM tip sliding processes 38 . If the system returns to the same state after the cycle, the free energy difference reads $\Delta \mathcal{F}=0$, and according to Eq. 19 the heat production can be evaluated simply as $\Delta q_{i}=T \Delta_{i} s=\Delta w$. However, due to the presence of thermal fluctuations, the cyclic free energy difference becomes a fluctuating quantity as well, and consequently $\Delta \mathcal{F} \neq 0$ after the cycle, and $\Delta q_{i} \neq \Delta w$. The valid approach then is to average the measurements over many 
repeated cycles, which leads to $\langle\Delta \mathcal{F}\rangle \rightarrow 0$ and consequently $\left\langle\Delta q_{i}\right\rangle \rightarrow\langle\Delta w\rangle$. Thus it is fundamentally possible to estimate only the mean value of the fluctuating heat production in the cyclic nanoscale friction experiment. The cyclic measurement can be compared with the measurement protocol based on the equilibrium starting and end points discussed throughout this article. The benefit of the present approach is that it allows analysing not only the heat production averages, but the fluctuations of heat around the average can be evaluated by means of Eq. (19) as well. It thus becomes possible to study rare extreme heat events that might have detrimental effects on the material sample.

\section{VELOCITY DEPENDENCE OF FRICTION AND HEAT}

In the following we apply the developed heat estimation procedure to practical calculations of velocity dependence of friction. Several asymptotic expressions relating the friction force to the FFM tip sliding velocity $V$ have been identified previously. In the thermal drift regime, when thermal fluctuations dominate the dynamics in the limit $V / a<\omega_{\mathrm{TD}}$ 24, a linear relation $\bar{f} \sim V$ was found in 33 and later extended to $\bar{f} \sim V^{2 / 3}$ in 34. In the above, $V / a$ is the characteristic (inverse) timescale of the sliding motion, and $\omega_{\mathrm{TD}}=\omega_{0} \exp \left(-\beta E_{0}\right)$ is the estimate of the smallest characteristic frequency of thermal fluctuations according to Eq. (3). We denoted by $\bar{f}$ the force averaged over the fluctuating trajectory as $\bar{f}=\Delta w / \Delta X$, where $\Delta X$ is the sliding length, to distinguish it from the fluctuating force $f$ used throughout this article.

In the intermediate velocity range where $V / a>\omega_{T D}$, corresponding to thermal stick-slip regime when both the dynamics due to the dragging forces and thermal fluctuations play a role, a logarithmic law $\bar{f} \sim \ln \left(V / V_{0}\right)$ was observed in 12]. This relation was later extended to $\bar{f} \sim\left|\ln \left(V / V_{0}\right)\right|^{2 / 3}$ applicable in a broader velocity range [31. These asymptotic relations fail when the velocity increases towards the non-thermal stick-slip regime in the limit $V / a>\omega_{0}$, when thermal fluctuations no longer play a role and the system dynamics is driven solely by the minimisation of energy in Eq. (1). Several forms have been derived to accommodate also this range within a unified framework. The implicit form $\left(f^{\star}-\bar{f}\right)^{3 / 2} / b=\ln \left(V_{0} / V\right)-\ln \left(1-\bar{f} / f^{\star}\right) / 2$ was found in [32], where $b, v_{0}$ are fit parameters and $f^{\star}=E_{0} \pi / a$. This relation is valid even at higher velocities up to several hundreds of $\mu \mathrm{m} / \mathrm{s}$. An alternative empirical form validated in a broad range of velocities and temperatures, which reads $\bar{f}=\sum_{\mathrm{n} \text { (odd) }} a_{n} \operatorname{arcsinh}\left(v / v_{c}\right)^{n}$ with $a_{n}$ and $v_{c}$ being fit parameters, was obtained in [35].

We have tested these relations based on our modelling approach over an extensive series of fluctuating trajectories used in Fig. 4, extended to a larger velocity range. Fig. 6(a) shows the comparison of the various formulas against the computed data set. The region of accuracy of
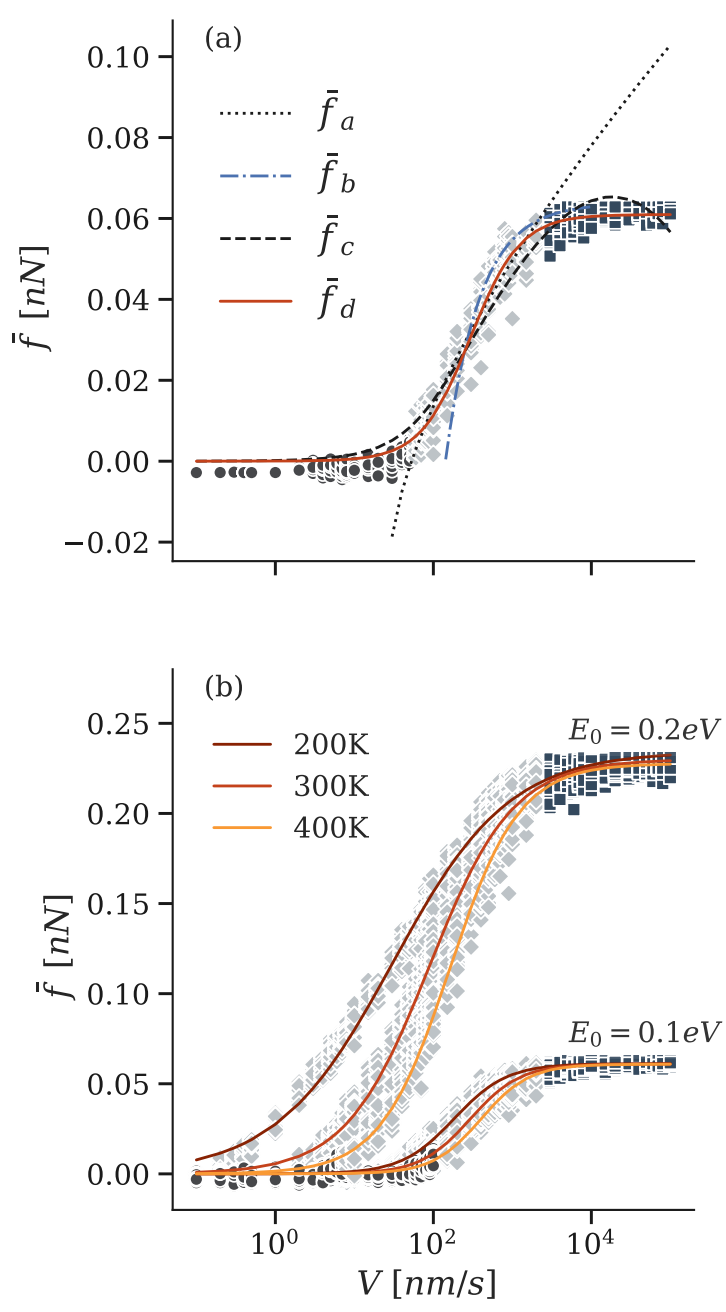

Figure 6. The mean friction force $\bar{f}$ versus the tip sliding velocity $V$. Each symbol corresponds to different fluctuating trajectory starting in equilibrium. (a) A comparison of accuracy of the least-squares fits of the force-velocity relations $\bar{f}_{a}=\alpha_{1}+\alpha_{2}\left|\ln \left(V / V_{0}\right)^{2 / 3}\right|$ derived in [31], the implicit formula $\left(f^{\star}-\bar{f}_{b}\right)^{3 / 2} / b=\ln \left(V_{0} / V\right)-\ln \left(1-\bar{f}_{b} / f^{\star}\right) / 2$ obtained in [32], the series expansion $\bar{f}_{c}=\sum_{n(\text { odd })=1}^{3} \alpha_{n} \operatorname{arcsinh}\left(v / v_{c}\right)^{n}$ from [35, and $\bar{f}_{d}$ calculated from Eq. 201. The different marker types represent the thermal drift, and thermal and non-thermal stick-slip friction regimes in the order of the increasing velocity. (b) Validation of Eq. 20 for a broad range of parameters corresponding to data in Fig. 4 but with extended velocity range. The fit parameter variations are discussed in the text.

the individual relations is clearly manifested showing that none of these relations covers the entire velocity range spanning the thermal drift and thermal and non-thermal stick-slip regimes. Therefore, we have experimented with various empirical functional forms ultimately identifying the following relation which describes the data with sat- 
isfactory statistical confidence:

$$
\bar{f}=\frac{L}{1+\left(V_{0} / V\right)^{r}} .
$$

Fig. 6 shows that this function indeed fits the entire data well. The constants $V_{0}, L$, are $r$ are fit parameters, that depend on the system parameters $E_{0}, C, a$, and on the temperature $T$. The parameter $L$ is the maximum asymptotic friction observable in the non-thermal high velocity range, only dependent on the non-thermal parameters of the model, i.e. $C, E_{0}$, and $a$. The parameter $V_{0}$ is the position of the middle (inflection) point of the curve. Fig. 6(b) shows further validation of the expression for different $E_{0}$ and $T$. We found $L$ is approximately $0.06 \mathrm{nN}$ for $E_{0}=0.1 \mathrm{eV}$, and $0.23 \mathrm{nN}$ for $E_{0}=0.2 \mathrm{eV}$. The parameter $r$ varies between $0.6-1.4$ and $V_{0}$ between $183-293 \mathrm{~nm} / \mathrm{s}$ for the individual data sets.

Eq. 20) seems valid in the full range of velocities studied. A series expansion of Eq. 20 around the mid-range velocity $V_{0}$ and its comparison with equivalent series expansion of the natural logarithm shows that $\bar{f}=L\left(1+\left(V_{0} / V\right)^{r}\right)^{-1} \approx L / 2+(r L / 4) \ln \left(V / V_{0}\right)$, which recovers the logarithmic dependence observed in the intermediate velocity range. Eq. 20 also compares well with the implicit form $f_{b}$ (Fig. 6(a) and series expansion $f_{c}$ (Fig. 6(a)), and in addition extends further to accommodate also the thermal drift regime. In the nonthermal stick-slip regime, Eq. 20 reproduces well the expected velocity independence of friction, also observed at the macroscale as Amontons' law.

Given the generic nature of Eq. (20), it is useful to link it with Eq. (19), which will allow obtaining the dissipative heat produced during the sliding process at a given velocity. This relation can be expressed as:

$$
\Delta q_{i}=\Delta \mathcal{F}-\bar{f} \Delta X
$$

where $\Delta q_{i}=T \Delta_{i} s$ as before, the fluctuating work was expressed through the mean fluctuating friction force acting over the length interval $\Delta X$, i.e. $\Delta w=\bar{f} \Delta X$ with $\bar{f}$ given by Eq. 20 . Thus, characterising a given material system through the friction force vs. velocity relation and identifying the equilibrium free energy difference $\Delta \mathcal{F}$ between the starting and final tip positions allows to quantitatively predict the heat dissipated during an arbitrary velocity friction process. In Sec. [V] we identified $\Delta \mathcal{F}$ based the fluctuation relations. The procedure relied on the repeated observations of the fluctuating work trajectories as input, which are observable also experimentally. However, since the number of trajectories required to achieve good statistics depended exponentially on the factor $\langle w\rangle / k_{B} T$, the approach may be practically limited to smaller values of $\langle w\rangle / k_{B} T$. Alternatively, $\Delta \mathcal{F}$ could be estimated by different means. For example, considering the measurements in Fig. 1 in [32, it would be plausible to assume that $\Delta \mathcal{F}$ is the same for every force vs. velocity scan curve at a given normal force. Applying Eq. 21) to the velocity scan curves individually then allows eliminating $\Delta \mathcal{F}$ from the equations, and thereby determining the relative heat changes at different velocities and normal force loads.

\section{CONCLUSION}

We demonstrated that the developed stochastic thermodynamics formalism is suitable for studying nanoscale friction in the stick-slip regime and allows interpreting the stochastic trajectories of tip observed in a typical FFM measurement in terms of basic thermodynamics concepts such as the fluctuating work and entropy. We validated the framework using the well-known Crooks and Jarzynski fluctuation relations. We then used these relations to determine the equilibrium free energy $\Delta \mathcal{F}$ from repeated measurements of the fluctuating work observable in a FFM experiment, and demonstrated that once $\Delta \mathcal{F}$ is known the associated fluctuating entropy (heat) production can be identified through a simple linear relation (Fig. 5). This allows interpreting a typical FFM measurement not only via the friction force, directly related to the work performed during the frictional sliding process, but also in terms of the heat produced during the process, which is an unambiguous measure of thermodynamic irreversibility. We have applied this procedure to quantify the velocity dependence of friction, where we succeeded in identifying empirical scaling relation encompassing the thermal drift, thermal stick-slip, and non-thermal stick-slip regimes relevant at different tip velocities, which complements the existing family of such relations discovered earlier.

The developed formalism can be naturally extended to two-dimensional surfaces by identifying the distributions of relevant energy barriers, which can provide new insight into how the material surface symmetries and commensurability determine the frictional sliding directions with minimal heat dissipation. The approach can be combined with ab initio methods to identify and substitute our Eq. (1) with potential energy surfaces of realistic materials, which will allow bottom-up design of materials with optimal control tailored for specific tribological applications.

\section{ACKNOWLEDGMENTS}

This work is supported by H2020 MSCA ITN project Solution No. 721642.
* Email: pc.torche@soton.ac.uk
[1] C. M. Mate, Tribology on the small scale: a bottom up approach to friction, lubrication, and wear, 6 (Oxford Uni- 
versity Press, 2008).

[2] S. R. De Groot and P. Mazur, Non-equilibrium thermodynamics (Courier Corporation, 2013).

[3] R. Zwanzig, Nonequilibrium statistical mechanics (Oxford University Press, 2001).

[4] U. Seifert, Reports on Progress in Physics 75, 126001 (2012).

[5] C. Van den Broeck and M. Esposito, Physica A: Statistical Mechanics and its Applications 418, 6 (2015).

[6] T. Tomé and M. J. de Oliveira, Physical Review Letters 108, 020601 (2012), publisher: American Physical Society.

[7] I. Barel, M. Urbakh, L. Jansen, and A. Schirmeisen, Tribology Letters 39, 311 (2010).

[8] A. Schirmeisen, L. Jansen, H. Hölscher, and H. Fuchs, Applied Physics Letters 88, 123108 (2006).

[9] E. Riedo and E. Gnecco, Nanotechnology 15, S288 (2004).

[10] S. Sills and R. M. Overney, Physical Review Letters 91, 095501 (2003), publisher: American Physical Society.

[11] R. Prioli, A. M. F. Rivas, F. L. Freire, and A. O. Caride, Applied Physics A: Materials Science and Processing 76, $565(2003)$

[12] E. Gnecco, R. Bennewitz, T. Gyalog, C. Loppacher, M. Bammerlin, E. Meyer, and H. J. Güntherodt, Physical Review Letters 84, 1172 (2000)

[13] L. Jansen, H. Hölscher, H. Fuchs, and A. Schirmeisen, Phys. Rev. Lett. 104, 256101 (2010).

[14] R. Bennewitz, R. Bennewitz, E. Gnecco, E. Gnecco, T. Gyalog, T. Gyalog, E. Meyer, and E. Meyer, Tribology Letters 10, 51 (2001)

[15] D. Gourdon, N. A. Burnham, A. Kulik, E. Dupas, D. Stamou, M. Liley, H. Vogel, C. Duschl, F. Oulevey, G. Gremaud, Z. Dienes, and Others, Trib. Lett. 3, 317 (1997)

[16] T. Onodera, Y. Morita, A. Suzuki, M. Koyama, H. Tsuboi, N. Hatakeyama, A. Endou, H. Takaba, M. Kubo, F. Dassenoy, C. Minfray, L. Joly-Pottuz, J.M. Martin, and A. Miyamoto, The Journal of Physical Chemistry B 113, 16526 (2009)

[17] T. Liang, S. R. Phillpot, and S. B. Sinnott, Phys. Rev. B 79, 245110 (2009)

[18] L. Prandtl, Journal of Applied Mathematics and Mechanics 8, 85 (1928).

[19] G. A. Tomlinson, The London, Edinburgh, and Dublin Philosophical Magazine and Journal of Science 7, 905 (1929)
[20] O. M. Braun and Y. S. Kivshar, Physics Reports 306, 1 (1998).

[21] Z.-J. Wang, T.-B. Ma, Y.-Z. Hu, L. Xu, and H. Wang, Friction 3, 170 (2015)

[22] F. Pellegrini, E. Panizon, G. E. Santoro, and E. Tosatti, Physical Review B 99, 075428 (2019)

[23] P. C. Torche, T. Polcar, and O. Hovorka, Phys. Rev. B 100, 125431 (2019).

[24] S. Y. Krylov and J. W. M. Frenken, Journal of Physics: Condensed Matter 20, 354003 (2008)

[25] M. Amiri and M. M. Khonsari, Entropy 12, 1021 (2010),

[26] R. Berkovich, J. Klafter, and M. Urbakh, Journal of Physics: Condensed Matter 20, 354008 (2008)

27] J. R. Gomez-Solano, C. July, J. Mehl, and C. Bechinger, New Journal of Physics 17, 045026 (2015)

[28] K. Sekimoto, Progress of Theoretical Physics Supplement 130, 17 (1998)

[29] G. E. Crooks, Physical Review E 60, 2721 (1999)

[30] C. Jarzynski, Physical Review E 56, 5018 (1997)

[31] Y. Sang, M. Dube, and M. Grant, Physical Review Letters 87, 174301 (2001), publisher: American Physical Society.

[32] E. Riedo, E. Gnecco, R. Bennewitz, E. Meyer, and H. Brune, Physical Review Letters 91, 084502 (2003), publisher: American Physical Society.

[33] S. Y. Krylov, K. B. Jinesh, H. Valk, M. Dienwiebel, and J. W. M. Frenken, Physical Review. E, Statistical, Nonlinear, and Soft Matter Physics 71, 065101 (2005).

[34] C. Fusco and A. Fasolino, Physical Review B 71, 045413 (2005)

[35] M. H. Müser, Physical Review B 84, 125419 (2011)

[36] Y. Dong, A. Vadakkepatt, and A. Martini, Tribology Letters 44, 367 (2011)

[37] C. M. Mate, G. M. McClelland, R. Erlandsson, and S. Chiang, Phys. Rev. Lett. 59, 1942 (1987)

[38] S. Y. Krylov and J. W. M. Frenken, physica status solidi (b) 251, 711 (2014).

[39] E. Gnecco, R. Roth, and A. Baratoff, Physical Review B 86, 035443 (2012), publisher: American Physical Society.

[40] M. C. Seiler and F. A. Seiler, Risk Analysis 9, 415 (1989)

[41] A. P. J. Jansen, An Introduction to Kinetic Monte Carlo Simulations of Surface Reactions (Springer, 2012).

[42] M. Evstigneev, J. J. Mazo, and P. Reimann, in Fundamentals of Friction and Wear on the Nanoscale (Springer, 2015) pp. 115-137.

[43] J. Schnakenberg, Reviews of Modern Physics 48, 571 (1976) 